\title{
Epidemiology, species distribution, and outcome of nosocomial Candida spp. bloodstream infection in Shanghai: an 11-year retrospective analysis in a tertiary care hospital
}

\author{
Yan-Jun Zheng ${ }^{1 \dagger}$, Ting Xie ${ }^{2 \dagger}$, Lin Wu ${ }^{3}$, Xiao-Ying Liư ${ }^{4}$, Ling Zhư ${ }^{5}$, Ying Chen ${ }^{1}$, En-Qiang Mao ${ }^{1}$, Li-Zhong Han ${ }^{6}$,
} Er-Zhen Chen ${ }^{1 * \dagger}$ and Zhi-Tao Yang ${ }^{1,7^{*+}+(1)}$

\begin{abstract}
Background: The incidence of Candida bloodstream infections (BSIs), has increased over time. In this study, we aimed to describe the current epidemiology of Candida BSI in a large tertiary care hospital in Shanghai and to determine the risk factors of 28-day mortality and the impact of antifungal therapy on clinical outcomes.

Methods: All consecutive adult inpatients with Candida BSI at Ruijin Hospital between January 1, 2008, and December 31, 2018, were enrolled. Underlying diseases, clinical severity, species distribution, antifungal therapy, and their impact on the outcomes were analyzed.

Results: Among the 370 inpatients with 393 consecutive episodes of Candida BSI, the incidence of nosocomial Candida BSI was 0.39 episodes/1000 hospitalized patients. Of the 393 cases, 299 (76.1\%) were treated with antifungal therapy (247 and 52 were treated with early appropriate and targeted antifungal therapy, respectively). The overall 28 -day mortality rate was $28.5 \%$, which was significantly lower in those who received early appropriate $(25.5 \%)$ or targeted (23.1\%) antifungal therapy than in those who did not (39.4\%; $P=0.012$ and $P=0.046$, respectively). In multivariate Cox regression analysis, age, chronic renal failure, mechanical ventilation, and severe neutropenia were found to be independent risk factors of the 28-day mortality rate. Patients who received antifungal therapy had a lower mortality risk than did those who did not.
\end{abstract}

Conclusions: The incidence of Candida BSI has increased steadily in the past 11 years at our tertiary care hospital in Shanghai. Antifungal therapy influenced short-term survival, but no significant difference in mortality was observed between patients who received early appropriate and targeted antifungal therapy.

Keywords: Candida spp., Bloodstream infection, Epidemiology, Species distribution, Antifungal therapy, Early treatment

*Correspondence: rjchenerzhen@163.com; yangzhitao@hotmail.fr †Yan-Jun Zheng, Ting Xie, Er-Zhen Chen, and Zhi-Tao Yang contributed equally to this manuscript

${ }^{1}$ Department of Emergency, Ruijin Hospital, Shanghai Jiao Tong University School of Medicine, Shanghai 200025, China

Full list of author information is available at the end of the article

\section{Background}

The incidence of invasive fungal infection has increased over time, especially for Candida bloodstream infections (BSIs), which is associated with considerable excess mortality and costs [1-3]. In the past two decades, the incidence of fungal infection has increased from 0.1 episodes/1000 admissions to $0.3-0.6$ episodes/1000

c) The Author(s) 2021. This article is licensed under a Creative Commons Attribution 4.0 International License, which permits use, sharing, adaptation, distribution and reproduction in any medium or format, as long as you give appropriate credit to the original author(s) and the source, provide a link to the Creative Commons licence, and indicate if changes were made. The images or other third party material in this article are included in the article's Creative Commons licence, unless indicated otherwise in a credit line to the material. If material is not included in the article's Creative Commons licence and your intended use is not permitted by statutory regulation or exceeds the permitted use, you will need to obtain permission directly from the copyright holder. To view a copy of this licence, visit http://creativeco mmons.org/licenses/by/4.0/. The Creative Commons Public Domain Dedication waiver (http://creativecommons.org/publicdomain/ zero/1.0/) applies to the data made available in this article, unless otherwise stated in a credit line to the data. 
admissions in China, North America, and several European countries [4-7]. The candida BSI mortality rate ranges from 35 to $53 \%$ [8-11]. The optimal management of Candida BSIs includes early awareness of patients at risk, control of the infection source, and timely administration of appropriate antifungal agents [12,13]. Consequently, antifungal agents have been widely used as empirical therapy. However, the overuse of antifungal agents results in increased costs, toxicity, and ecological selection pressure for antifungal resistance and adverse drug interactions. Several studies showed that delayed antifungal therapy (more than $48 \mathrm{~h}$ from onset) is associated with higher mortality [14], whereas others have reported conflicting results [15-17].

In this study, we retrospectively analyzed data from all patients with Candida BSI at our hospital between 2008 and 2018, aiming to describe their clinical characteristics, species distribution, antifungal therapy and to determine the risk factors for 28-day mortality.

\section{Methods}

\section{Study setting and population}

A retrospective analysis of data on consecutive Candida BSI episodes in adult inpatients ( $\geq 18$ years) between January 1,2008 , and December 31, 2018, collected from the microbiology database of a 1900-bed teaching hospital in Shanghai, was performed.

Demographics and data on underlying diseases, comorbidities, severity of clinical features, Candida species distribution, and early appropriate or targeted antifungal treatment were compared among the patients with Candida BSI. Data on the initial and targeted antifungal agents used were also collected.

For each patient, aerobic and anaerobic blood culture bottles were each inoculated with $10 \mathrm{ml}$ of blood and transported to the laboratory within $1 \mathrm{~h}$ of collection. An episode of candidemia was defined as the first isolation (incident candidemia) of Candida species from blood culture in a patient with signs of infection. Blood cultures of patients with long lines (central venous catheter (CVC), Hickman or PICC) were taken through both peripheral vein and longlines, instances where longlines blood culture were positive but the peripheral blood culture remained negative indicate colonization of the catheter rather than BSI. New positive blood cultures within 30 days from the incident candidemia were considered part of the same episode [18]. BSI was considered as nosocomial if diagnosed at least $48 \mathrm{~h}$ after hospital admission $[19,20]$. We evaluated the clinical manifestations and host factors when confirming whether it was genuine candidemia. Severe neutropenia was defined as $<500 / \mathrm{mm}^{3}$ absolute neutrophil count. Prior corticosteroid was defined as receiving $>1$ $\mathrm{mg} / \mathrm{kg} / \mathrm{d}$ prednisone for more than one week or equivalent before Candida BSI onset.

\section{Laboratory methods}

Isolates were detected from blood cultures using the BACTEC $^{\mathrm{TM}}$ FX system (Becton Dickinson, Inc., Sparks, MD, USA), identified using VITEK-2 system (bioMérieux, Marcy-l'Étoile, France) before 2015, and matrix-assisted laser desorption ionization-time of flight mass spectrometer (bioMérieux, Marcy-l'Étoile, France) after 2015. Susceptibility testing for flucytosine, amphotericin B, fluconazole, voriconazole, and itraconazole was performed using the $\mathrm{ATB}^{\circledR}{ }^{\circledR}$ FUNGUS 3 system (BioMérieux, France), which is widely used in China [21]. This test provides information on susceptibility to antifungals agents, which is concordant with that obtained using the methodologies of the Clinical and Laboratory Standards Institute (CLSI) and European Committee on Antimicrobial Susceptibility Testing (EUCAST) [22].

Early appropriate antifungal treatment was defined as commencement of appropriate drug treatment at an adequate dosage before obtaining in vitro susceptibility test results. The adequate dosage of the antifungal agent was defined according to 2009 or 2016 Infectious Diseases Society of America (IDSA) guidelines [18, 23]. Targeted antifungal treatment was defined as commencement of appropriate targeted treatment after obtaining results from susceptibility testing, regardless of whether the antifungal treatment initiated was appropriate. Crude mortality was calculated from data on deaths registered 28 days after the occurrence of Candida BSI.

\section{Statistical analysis}

Descriptive and subgroup analyses were performed for the baseline characteristics, and continuous variables were expressed as mean \pm standard deviation (SD) or median and interquartile range according to their distributions. The chi-square test or 2-tailed Fisher exact test was applied to categorical variables. To identify the risk factors for 28-day mortality, multivariate Cox regression analysis was performed, and adjusted hazard ratio (HRs) with $95 \%$ confidence intervals (CIs) were reported. Variables that were associated with 28-day mortality in the Cox univariate analyses with a $\mathrm{P}<0.05$ were entered into the multivariate Cox regression analysis model based on the forward selection. Two-tailed tests of significance at the level of a $\mathrm{P}$ value $<0.05$ level was considered as significant. Statistical analysis was performed using IBM SPSS Statistics for Windows, version 22.0 (IBM Corp., Armonk, N.Y. USA). 


\section{Ethics}

The study was approved by Ruijin Hospital, Shanghai Jiao Tong University, School of Medicine institutional review board, and written informed consent was not required because of the observational nature of this study.

\section{Results}

Incidence and clinical features of Candida BSI episodes

Data on a total of 393 consecutive episodes of Candida BSI were collected from 370 inpatients during the 11-year study period. No outbreaks were reported during this period. The demographic characteristics of the patients are summarized in Table 1. The mean age of the patients was $57.6 \pm 19.0$ years, and $74.3 \%$ were male. Candida BSI incidence was 0.39 episodes/1000 admissions. The incidence increased steadily from 0.21 (2008), to 0.59 (2017), to 0.33 episodes per 1,000 admissions (2018) (Fig. 1a). Among the 393 Candida BSI episodes, 148 (37.7\%), 167 (42.5\%), and 78 (19.8\%) occurred in the surgical ward, intensive care units (ICUs), and internal medicine ward, respectively (Fig. 1b).

C. albicans was isolated in 19.3, 41.2, and 38.9\%, of cases in internal medicine wards, surgery wards, and the ICU, respectively $(\mathrm{P}=0.003)$. A higher proportion of $C$. tropicalis $(34.7 \%)$ was found in internal medicine wards than in the surgery wards (21.6\%) and ICUs (21.6\%).

Most patients with Candida BSI had at least one comorbidity. These included 118 (30\%) patients with solid tumors, 48 (12.2\%) had hematological malignancies, 77 (19.6\%) had diabetes mellitus, 124 (31.6\%) had chronic cardiac disease, 52 (13.2\%) had chronic pulmonary disease, 42 (10.7\%) had chronic renal failure, in 26 (6.6\%) patients, the skin barrier was considered compromised, $244(62.1 \%)$ had prior surgical intervention, 54 (13.7\%) used corticosteroid, 88 (22.4\%) used prior antifungal agents, and 255 (64.9\%) received antibiotics prior Candida BSI onset. A total of 244 (72\%) patients had at least two comorbidities. Regarding the severity, 309 (78.6\%) patients had fever, $180(45.8 \%)$ received parenteral nutrition, 147 (37.4\%) received mechanical ventilation, 49 $(12.5 \%)$ received renal replacement therapy, and 42 (10.7\%) had severe neutropenia. The clinical characteristics of patients by Candida species are shown in Table 1.

\section{Antifungal susceptibility of Candida isolates}

A total of 393 Candida spp. were isolated, including 141 (35.9\%), C. albicans, 87, C. parapsilosis (22.1\%); 69, C. tropicalis $(17.6 \%) ; 48$, C. glabrata $(12.2 \%) ; 20$, C. guilliermondii (5.1\%); 8, C. sake (2.0\%); 5, C.krusei (1.3\%); and 15, other species (4, C. gum; 3, C. lusitaniae; 2, C. intermedia; 2, C. theae; 2, C. lipolytica; 1, C. famata; and 1, C. haemulonii).

Among the 393 Candida species, 378 were subjected to antifungal susceptibility testing, based on 2012 CLSI breakpoints (CBPs) [24]. As shown in Table 2, the susceptibility of C. albicans, C. parapsilosis to fluconazole, and voriconazole were quite high, compared to that of itraconazole (94, 93.3 vs. $82.1 \%)$. The susceptibility of $C$. tropicalis to triazoles fluconazole, voriconazole, and itraconazole was unsatisfactory. Amphotericin B and 5-flucytosine remained superior against common Candida spp., except for C. krusei and C. guilliermondii, with 95\% susceptibility.

\section{Antifungal therapy and outcome of patients with Candida BSI}

Antifungal therapy was administered to 299 (76.1\%) patients, whereas 94 (23.9\%) patients did not receive antifungal therapy. The Comparison between the patients receiving antifungal therapy and those without antifungal therapy was shown in Table 3 . Among those who received antifungal therapy, 247 (62.8\%) received early appropriate antifungal therapy, and 52 (13.2\%) received targeted antifungal therapy. Fluconazole was most frequently used as empirical therapy, followed by echinocandins and voriconazole. Eighteen (4.6\%) patients with Candida BSI received combination therapy.

The overall, 28 -day mortality rate was $28.5 \%$, and the rate was significantly higher in internal medicine wards and ICUs than in surgical wards $(37.2 \%$ and $34.7 \%$ vs. $16.9 \%$, respectively, $\mathrm{P}<0.001$ ). As shown in Fig. 2 , the mortality rates among those who received early appropriate or targeted antifungal therapy were $26.8 \%$ and $25.1 \%(\mathrm{P}=0.012$ or $\mathrm{P}=0.046)$, compared to $39.3 \%$ for those who did not receive any antifungal therapy, with no significant difference $(\mathrm{P}=0.732)$ between those who received early appropriate antifungal therapy and those who received targeted antifungal therapy.

On univariate analysis, age, solid tumor, diabetes mellitus, chronic cardiac disease, chronic renal failure, skin disease, prior surgical intervention, mechanical ventilation, severe neutropenia, and antifungal therapy were found to be associated with 28-day mortality. On multivariate Cox regression analysis, advanced age $(\mathrm{HR}=1.025 ; 95 \% \mathrm{CI}, 1.013-1.037 ; \mathrm{P}<0.001)$, chronic renal failure $(H R=2.018 ; 95 \% C I$ 1.234-3.299; $\mathrm{P}=0.005)$, mechanical ventilation $(\mathrm{HR}=1.950$; 95\%CI 1.307-2.912; $\mathrm{P}=0.001)$, and severe neutropenia $(\mathrm{HR}=4.347 ; 95 \% \mathrm{CI}$ 2.462-7.675; $\mathrm{P}<0.001$ ), were found to be independent risk factors for 28-day mortality. However, antifungal therapy $(\mathrm{HR}=0.570 ; 95 \% \mathrm{CI} 0.382-0.849 ; \mathrm{P}=0.006)$ was an independent protective factor for 28-day mortality (Table 4). 
Table 1 Demographic and clinical data for patients with Candida bloodstream infection

\begin{tabular}{|c|c|c|c|c|c|c|c|c|c|}
\hline & $\begin{array}{l}\text { C. albicans } \\
(n=141)\end{array}$ & $\begin{array}{l}\text { C. parapsilosis } \\
(n=87)\end{array}$ & $\begin{array}{l}\text { C. tropicalis } \\
(n=69)\end{array}$ & $\begin{array}{l}\text { C. glabrata } \\
(n=48)\end{array}$ & $\begin{array}{l}\text { C. } \\
\text { guilliermondii } \\
(\mathrm{n}=20)\end{array}$ & $\begin{array}{l}C \\
\text { sake } \\
(n=8)\end{array}$ & $\begin{array}{l}C \\
\text { krusei } \\
(n=5)\end{array}$ & $\begin{array}{l}\text { Other Candida } \\
\text { spp. } \\
(n=15)\end{array}$ & $\begin{array}{l}\text { Total } \\
(n=393)\end{array}$ \\
\hline Age, years & $65.2 \pm 14.5$ & $53.2 \pm 20.3$ & $50.5 \pm 19.5$ & $60.7 \pm 17.5$ & $50.5 \pm 19.4$ & $52 \pm 20.6$ & $40.8 \pm 27.1$ & $52.6 \pm 20.0$ & $57.6 \pm 19.0$ \\
\hline Male & $102(73.4)$ & $60(69.0)$ & $51(73.9)$ & $44(91.7)$ & $14(70)$ & $8(100)$ & $2(40)$ & $11(73.3)$ & $292(74.3)$ \\
\hline \multicolumn{10}{|l|}{ Origin } \\
\hline $\begin{array}{l}\text { Internal medicine } \\
\text { ward }\end{array}$ & $15(10.6)$ & $19(21.8)$ & $27(39.1)$ & $7(14.6)$ & $3(15)$ & $0(0)$ & $3(60)$ & $4(26.7)$ & 78(19.8) \\
\hline Surgical ward & $61(43.3)$ & $32(36.8)$ & $16(23.2)$ & $15(31.3)$ & $10(50)$ & $6(75)$ & $0(0)$ & $8(53.3)$ & 148(37.7) \\
\hline ICU & $65(46.1)$ & $36(41.4)$ & $26(37.7)$ & $26(54.1)$ & $7(35)$ & $2(25)$ & $2(40)$ & $3(20)$ & $167(42.5)$ \\
\hline $\begin{array}{l}\text { Time from admis- } \\
\text { sion to infection, } \\
\text { days }\end{array}$ & $30.6 \pm 35.3$ & $48.4 \pm 56.2$ & $37.7 \pm 32.4$ & $27.5 \pm 19.1$ & $35.9 \pm 52.5$ & $120.6 \pm 242.7$ & $64.2 \pm 68.6$ & $21.5 \pm 13.6$ & $37.6 \pm 53.1$ \\
\hline $\begin{array}{l}\text { Length of hospital } \\
\text { stay, days }\end{array}$ & $56.0 \pm 54.9$ & $83.2 \pm 78.1$ & $71.5 \pm 56.1$ & $67.1 \pm 72.7$ & $97.7 \pm 142.6$ & $178.5 \pm 281.7$ & $92.4 \pm 88.5$ & $52.7 \pm 101.5$ & $71.1 \pm 82.8$ \\
\hline $\begin{array}{l}\text { Turnaround time, } \\
\text { days }\end{array}$ & $4.3 \pm 1.9$ & $4.5 \pm 1.1$ & $3.8 \pm 1.1$ & $4.8 \pm 1.3$ & $4.3 \pm 1.2$ & $4.5 \pm 1.6$ & $4.4 \pm 1.5$ & $5.9 \pm 1.8$ & $4.4 \pm 1.5$ \\
\hline \multicolumn{10}{|l|}{ Underlying disease } \\
\hline Solid tumor & $47(33.3)$ & 26(29.9) & 13(18.8) & $17(35.4)$ & $6(30)$ & $2(25)$ & $0(0)$ & $7(46.7)$ & 118(30) \\
\hline $\begin{array}{l}\text { Hematologic } \\
\text { malignancy }\end{array}$ & $8(5.7)$ & $6(6.9)$ & 24(34.8) & $2(4.2)$ & $2(10)$ & $1(12.5)$ & $3(60)$ & $2(13.3)$ & $48(12.2)$ \\
\hline Diabetes mellitus & $33(23.4)$ & $21(24.1)$ & $8(11.6)$ & $9(18.8)$ & $1(5)$ & $1(12.5)$ & $0(0)$ & $4(26.7)$ & $77(19.6)$ \\
\hline $\begin{array}{l}\text { Chronic cardiac } \\
\text { disease }\end{array}$ & $55(39)$ & $22(25.3)$ & $16(23.2)$ & $18(37.5)$ & $6(30)$ & $3(37.5)$ & $2(40)$ & $2(13.3)$ & 124(31.6) \\
\hline $\begin{array}{l}\text { Chronic pulmo- } \\
\text { nary disease }\end{array}$ & $26(18.4)$ & $9(10.3)$ & $5(7.2)$ & $6(12.5)$ & $1(5)$ & $1(12.5)$ & $1(20)$ & $3(20)$ & $52(13.2)$ \\
\hline $\begin{array}{l}\text { Chronic renal } \\
\text { failure }\end{array}$ & $18(12.8)$ & $6(6.9)$ & $6(8.7)$ & $5(10.4)$ & $5(25)$ & $0(0)$ & $0(0)$ & $2(13.3)$ & $42(10.7)$ \\
\hline $\begin{array}{l}\text { Skin barrier com- } \\
\text { promised }\end{array}$ & $5(3.5)$ & $9(10.3)$ & $5(7.2)$ & $1(2.1)$ & $3(15)$ & $3(37.5)$ & $0(0)$ & $0(0)$ & $26(6.6)$ \\
\hline $\begin{array}{l}\text { Prior surgical } \\
\text { intervention }(<1 \\
\text { month) }\end{array}$ & $97(68.8)$ & $48(55.2)$ & $36(52.2)$ & $30(62.5)$ & $15(75)$ & $6(75)$ & $2(40)$ & $10(66.7)$ & $244(62.1)$ \\
\hline Corticosteroid use & $11(7.8)$ & 13(14.9) & $12(17.4)$ & $9(18.8)$ & $4(20)$ & $0(0)$ & $3(60)$ & $2(13.3)$ & $54(13.7)$ \\
\hline $\begin{array}{l}\text { Prior use of } \\
\text { antifungal agents } \\
\text { (<6 months })\end{array}$ & 20(14.2) & 19(21.8) & 24(34.8) & $11(22.9)$ & $8(40)$ & $1(12.5)$ & $4(80)$ & $1(6.7)$ & $88(22.4)$ \\
\hline \multicolumn{10}{|c|}{ Severity of clinical feature } \\
\hline Fever $\left(\mathrm{T}>38.2^{\circ} \mathrm{C}\right)$ & 114(80.9) & $63(72.4)$ & $60(87)$ & $34(70.8)$ & $16(80)$ & $6(75)$ & $3(60)$ & 13(86.7) & $309(78.6)$ \\
\hline $\begin{array}{l}\text { Parenteral nutri- } \\
\text { tion }\end{array}$ & $71(50.4)$ & $42(48.3)$ & $28(40.6)$ & 23(47.9) & $5(25)$ & $3(37.5)$ & $2(40)$ & $6(40)$ & 180(45.8) \\
\hline $\begin{array}{l}\text { Mechanical venti- } \\
\text { lation }\end{array}$ & $58(41.1)$ & $31(35.6)$ & 23(33.3) & $25(52.1)$ & $5(25)$ & $2(25)$ & $2(40)$ & $1(6.7)$ & 147(37.4) \\
\hline $\begin{array}{l}\text { Renal replacement } \\
\text { therapy }\end{array}$ & 17(12.1) & $10(11.5)$ & $9(13)$ & $7(14.6)$ & $5(25)$ & $0(0)$ & $1(20)$ & $0(0)$ & $49(12.5)$ \\
\hline $\begin{array}{l}\text { Central venous } \\
\text { catheter }\end{array}$ & $121(85.8)$ & $67(77)$ & $49(71)$ & 43(89.6) & $17(85)$ & $5(62.5)$ & $4(80)$ & $10(66.7)$ & 316(80.4) \\
\hline Severe neutropenia & $4(2.8)$ & $8(9.2)$ & 22(31.9) & $1(2.1)$ & $2(10)$ & $0(0)$ & $3(60)$ & $2(13.3)$ & $42(10.7)$ \\
\hline 28-day mortality & $54(38.3)$ & $16(18.4)$ & $19(27.5)$ & $13(27.1)$ & $3(15)$ & $1(12.5)$ & $1(20)$ & $5(33.3)$ & $112(28.5)$ \\
\hline
\end{tabular}

Other Candida spp. Includes C. gum (4 cases), C. lusitaniae (3 cases), C. intermedia (2 cases), C. lipolytica (2cases), C. theae (2 cases), C.famata (1 case), and C. haemulonii (1 case)

Data were expressed as mean \pm SD for continuous variables and $\mathrm{n}(\%)$ for categorical variables ICU intensive care unit, SD standard deviation 
a Distribution of Candida species and episodes/1000 admissions during the study period

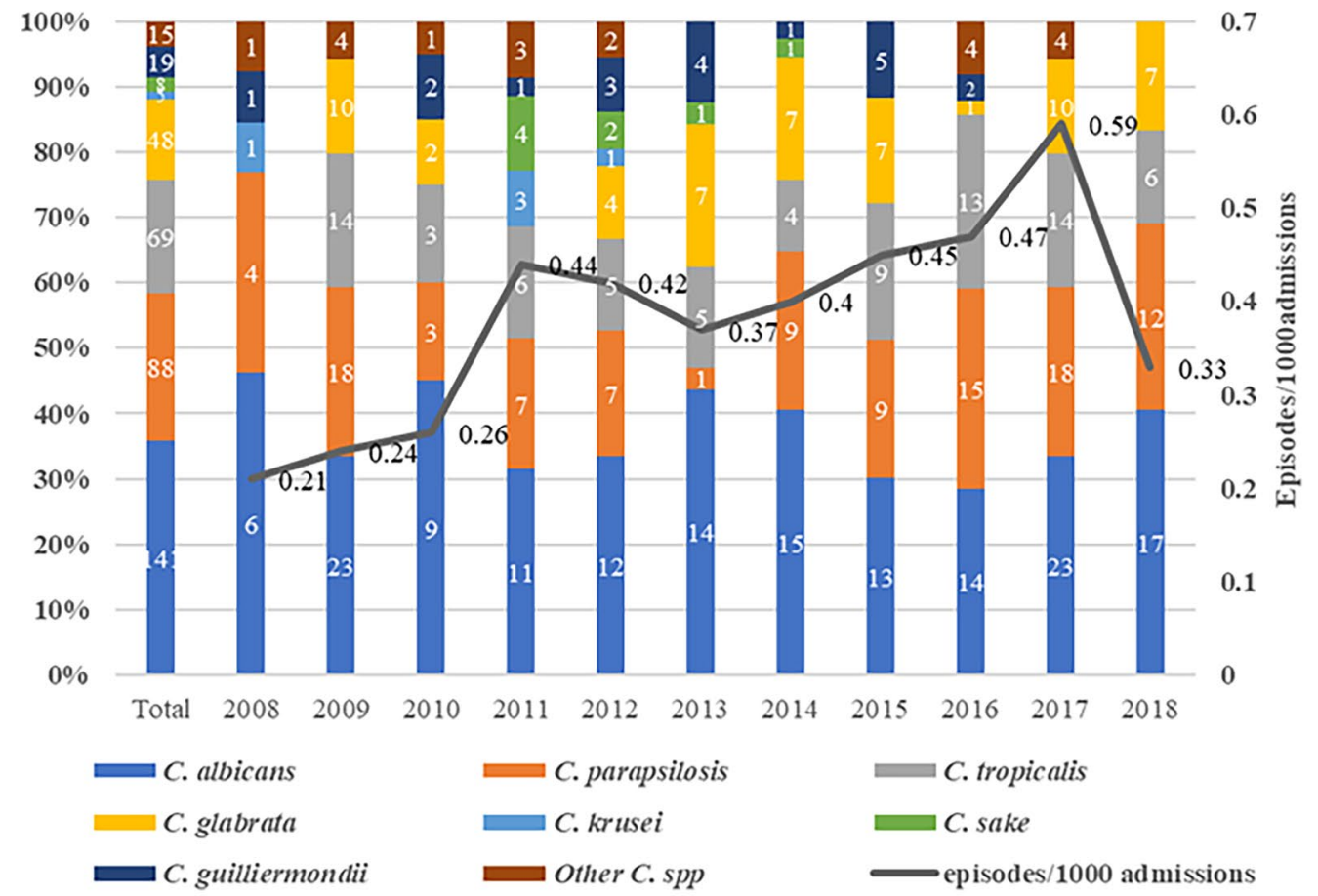

b Distribution of Candida species according to hospital wards

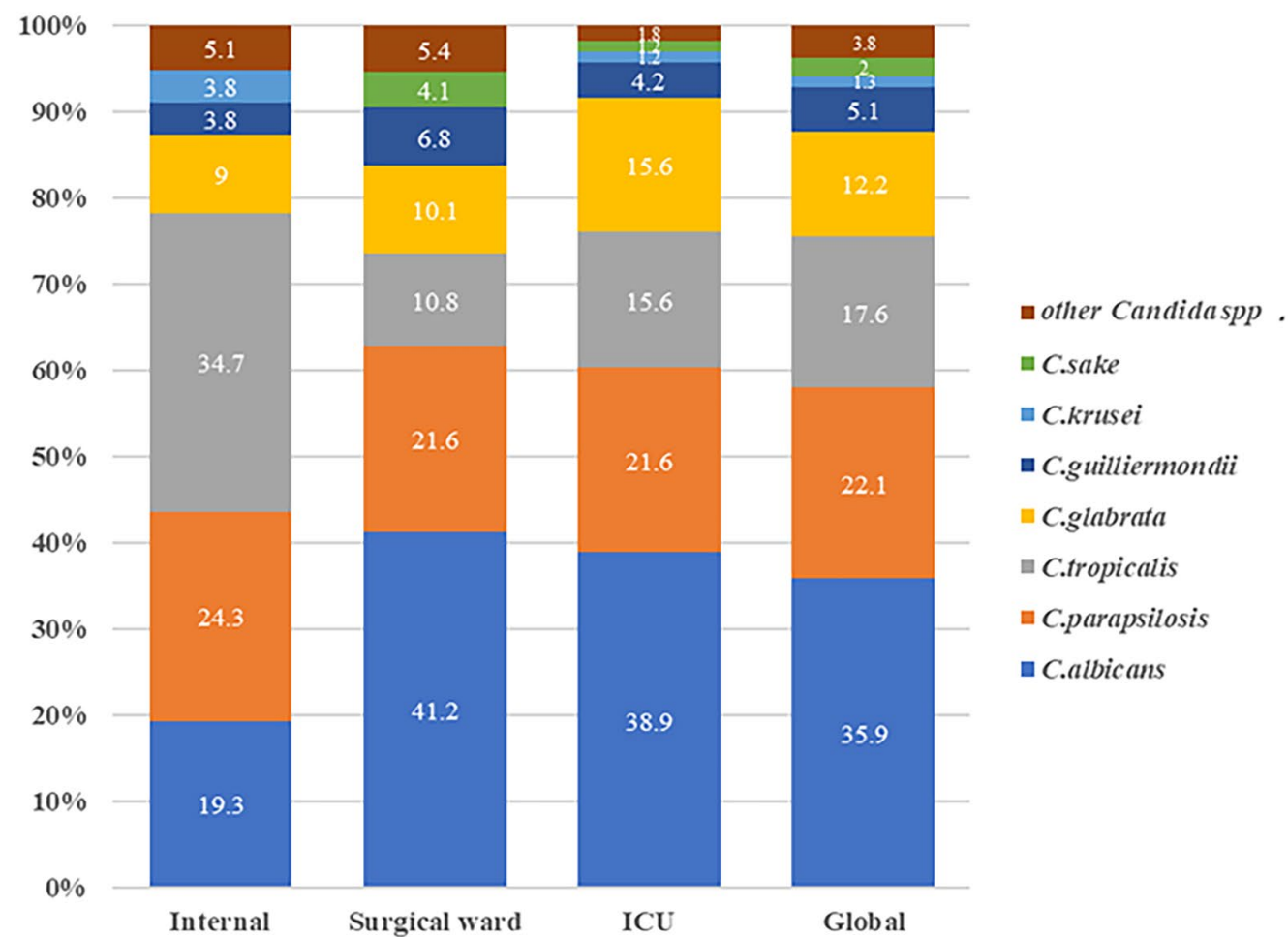

Fig. 1 a Distribution of Candida spp. and episodes/1000 admissions during the study period. b Distribution of Candida spp. according to hospital wards; 148 episodes were from surgical wards; 167 episodes were from ICUs and 78 episodes were from the internal medicine wards 
Table 2 Antifungal susceptibility testing results (ATB Fungus 3) of 378 Candida [n (\%)]

\begin{tabular}{|c|c|c|c|c|c|c|c|c|c|}
\hline & $\begin{array}{l}\text { C.albicans } \\
(n=134)\end{array}$ & $\begin{array}{l}\text { C. } \\
\text { parapsilosis } \\
(n=86)\end{array}$ & $\begin{array}{l}\text { C.tropicalis } \\
(\mathrm{n}=67)\end{array}$ & $\begin{array}{l}\text { C.glabrata } \\
(n=47)\end{array}$ & $\begin{array}{l}\text { C.krusei } \\
(n=5)\end{array}$ & $\begin{array}{l}\text { C.sake } \\
(\mathrm{n}=8)\end{array}$ & $\begin{array}{l}\text { C. } \\
\text { guilliermondii } \\
(n=19)\end{array}$ & $\begin{array}{l}\text { Other } \\
\text { Candida spp. } \\
(\mathrm{n}=12)\end{array}$ & Total $(\mathrm{n}=378)$ \\
\hline \multicolumn{10}{|c|}{ Fluconazole } \\
\hline S & $126(94)$ & 77(89.5) & $35(52.2)$ & $0(0)$ & $0(0)$ & $8(100)$ & $13(68.4)$ & $9(75.0)$ & 268 (70.9) \\
\hline SDD & $1(0.8)$ & $6(7.0)$ & $3(4.5)$ & $44(93.6)$ & $0(0)$ & $0(0)$ & $0(0)$ & $0(0)$ & $54(14.3)$ \\
\hline $\mathrm{R}$ & $7(5.2)$ & $3(3.5)$ & $29(43.3)$ & $3(6.4)$ & $5(100)$ & $0(0)$ & $6(31.6)$ & $3(25.0)$ & $56(14.8)$ \\
\hline \multicolumn{10}{|c|}{ Itraconazole } \\
\hline S & $110(82.1)$ & $75(87.2)$ & $25(37.3)$ & $0(0)$ & $0(0)$ & $8(100)$ & $6(31.6)$ & $9(75.0)$ & 233 (61.6) \\
\hline SDD & $6(4.5)$ & $7(8.1)$ & $4(6.0)$ & $40(85.1)$ & $2(40.0)$ & $0(0)$ & $7(36.8)$ & $0(0)$ & $66(17.5)$ \\
\hline $\mathrm{R}$ & $18(13.4)$ & $4(4.7)$ & $38(56.7)$ & $7(14.9)$ & $3(60.0)$ & $0(0)$ & $6(31.6)$ & $3(25.0)$ & 79 (20.9) \\
\hline \multicolumn{10}{|c|}{ Voriconazole } \\
\hline S & 125 (93.3) & 79 (91.9) & $41(61.2)$ & 45 (95.8) & $4(80.0)$ & $8(100)$ & $12(63.2)$ & $11(91.7)$ & $325(86.0)$ \\
\hline SDD & $0(0)$ & $2(2.3)$ & $2(3.0)$ & $1(2.1)$ & $1(2.0)$ & $0(0)$ & $3(15.8)$ & $0(0)$ & $9(2.4)$ \\
\hline $\mathrm{R}$ & $9(6.7)$ & $5(5.8)$ & $24(35.8)$ & $1(2.1)$ & $0(0)$ & $0(0)$ & $4(21.0)$ & $1(8.3)$ & $44(11.6)$ \\
\hline \multicolumn{10}{|c|}{ Amphotericin B } \\
\hline $\mathrm{S}$ & 133 (99.3) & $83(96.5)$ & $67(100)$ & $47(100)$ & $5(100)$ & $8(100)$ & 18 (94.7) & 11 (91.7) & $372(98.4)$ \\
\hline $\mathrm{R}$ & $1(0.7)$ & $3(3.5)$ & $0(0)$ & $0(0)$ & $0(0)$ & $0(0)$ & $1(5.3)$ & $1(8.3)$ & $6(1.6)$ \\
\hline \multicolumn{10}{|c|}{ Flucytosine } \\
\hline S & $132(98.5)$ & 85 (98.8) & $65(97.0)$ & $46(97.9)$ & $1(20.0)$ & $8(100)$ & 9 (47.4) & $12(100)$ & $358(94.7)$ \\
\hline R & $2(1.5)$ & $1(1.2)$ & $2(3.0)$ & $1(2.1)$ & $4(80.0)$ & $0(0)$ & $10(52.6)$ & $0(0)$ & $20(5.3)$ \\
\hline
\end{tabular}

15 Candida spp. isolates did not have a susceptibility test, C. albicans (7), C. parapsilosis (2), C. tropicalis (2), and glabrata, theae, gum, haemulonii each $R$ resistance, $S$ susceptible, SDD susceptible dose dependence

Table 3 Comparison between the patients receiving antifungal therapy and without antifungal therapy

\begin{tabular}{|c|c|c|c|}
\hline & Antifungal therapy $(n=299)$ & No antifungal treatment $(n=94)$ & $P$ value \\
\hline Age & $56.47 \pm 19.19$ & $61.13 \pm 17.81$ & 0.038 \\
\hline Male & $223(74.6)$ & $69(73.4)$ & 0.820 \\
\hline \multicolumn{4}{|l|}{ Underlying disease } \\
\hline Solid tumor & $80(26.8)$ & $38(40.4)$ & 0.012 \\
\hline Hematologic malignancy & $39(13.0)$ & $9(9.6)$ & 0.370 \\
\hline Diabetes mellitus & $56(18.7)$ & $21(22.3)$ & 0.442 \\
\hline Chronic Cardiac disease & $88(29.4)$ & $36(38.3)$ & 0.107 \\
\hline Chronic Pulmonary disease & $44(14.7)$ & $8(8.5)$ & 0.121 \\
\hline Chronic renal failure & 29(9.7) & 13(13.8) & 0.258 \\
\hline Skin barrier compromised & $21(7.0)$ & $5(5.3)$ & 0.016 \\
\hline Prior surgical intervention (<1 month) & 182(60.9) & $62(66.0)$ & 0.375 \\
\hline Corticosteroid use & $45(15.1)$ & $9(9.6)$ & 0.179 \\
\hline Prior antifungal agents use (<6 month) & $76(25.4)$ & $12(12.8)$ & 0.010 \\
\hline \multicolumn{4}{|l|}{ Severity of clinical feature } \\
\hline Fever $\left(\mathrm{T}>38.2^{\circ} \mathrm{C}\right)$ & $244(81.6)$ & $65(69.1)$ & 0.010 \\
\hline Parenteral nutrition & 135(45.2) & $45(47.9)$ & 0.644 \\
\hline Mechanical ventilation & 113(37.8) & $34(36.2)$ & 0.777 \\
\hline Renal replacement therapy & $36(12.0)$ & 13(13.8) & 0.647 \\
\hline Central venous catheter & $242(80.9)$ & $74(78.7)$ & 0.637 \\
\hline Severe neutropenia & $36(12.0)$ & $6(6.4)$ & 0.338 \\
\hline APACHE II score & $11.86 \pm 5.91$ & $11.72 \pm 5.37$ & 0.928 \\
\hline SOFA score & $3.66 \pm 3.56$ & $4.22 \pm 4.24$ & 0.577 \\
\hline
\end{tabular}




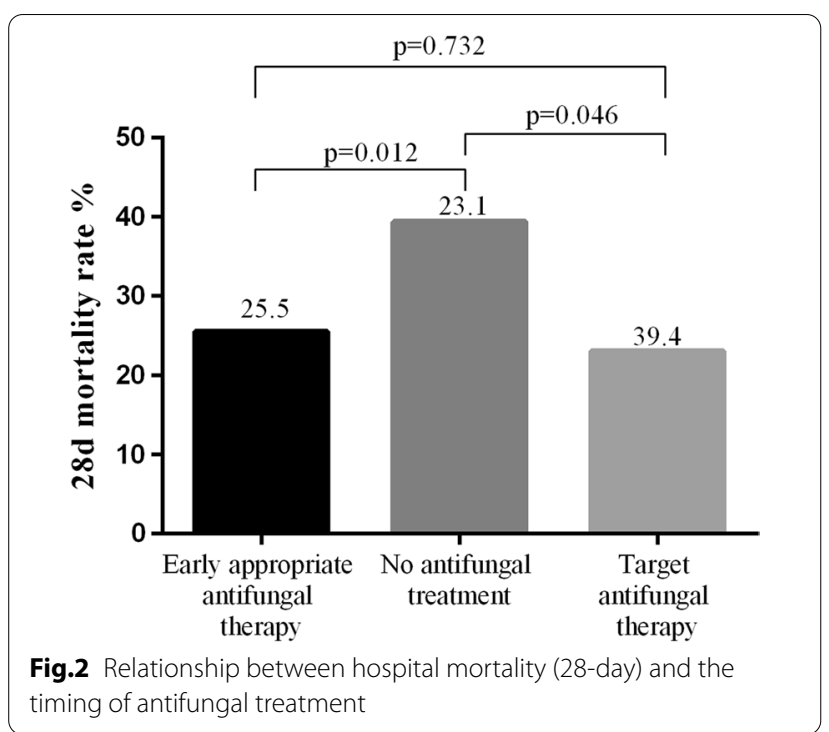

\section{Discussion}

Our study showed that the incidence of Candida BSI has increased steadily in the past 11 years at our tertiary care hospital in Shanghai. Several studies have shown a substantial increase in Candida BSI incidence in the past two decades, which is similar to our study findings $[4,11$, 25].

C. albicans remains the most common pathogen causing Candida BSI. However, over the past two decades, an increased percentage of common non-C. albicans Candida spp. have been reported worldwide. In our study, non-C. albicans accounted for $64.1 \%$. C. parapsilosis, C. tropicalis, and C. glabrata accounted for most of the nonC. albicans species. However, some studies in western countries showed that C. glabrata was the most frequent species among non-C. albicans, whereas C. tropicalis was quite rare compared to the incidence reported in our study (5.9 and 7.0 vs. $27.4 \%$ ) [26, 27]. In this study, the incidence of C. guilliermondii was significantly higher than that in other studies (4.8 vs. 0.4\%) [28].

The antifungal susceptibility testing showed that the susceptibility of C. albicans,

C. parapsilosis and C. sake to fluconazole were quite high $(94,89.5,100 \%)$. On the other hand, the resistance rate of C. tropicalis for fluconazole was as high as $43.3 \%$, which was significantly higher than those reported in other studies abroad [29, 30]. This may deserve more attention from clinicians in actual application. However, the antifungal results in our study were according to 2012 CLSI, since we didn't have the MIC values for the antifungals, we couldn't reanalyze according to new CLSI guidelines on antifungal susceptibility M60 published in 2017. The susceptibility testing in our study was performed using the $\mathrm{ATB}^{\circledR}$ FUNGUS 3 system, there were some contradictory views about its performance. Zhang $\mathrm{L}$ et al. thought $\mathrm{ATB}^{\circledR}$ FUNGUS 3 might misleading high MICs of Candida spp. to azoles[31], while some other studies support $A T B^{\circledR}$ FUNGUS system was an objective, reproducible and simple method for the accurate determination of MICs of the most common antifungal drugs in yeasts[32, 33].

In our hospital, the physicians administrated prophylactic antifungal therapy and empirical therapy according to the patient's risk factors and clinical manifestation. Fluconazole (800-mg loading dose, then $400 \mathrm{mg}$ daily) was the most used prophylactic antifungal agent during the study period. Fluconazole (800-mg loading dose, then $400 \mathrm{mg}$ daily) and caspofungin (loading dose of $70 \mathrm{mg}$, then $50 \mathrm{mg}$ daily) were the most used empirical therapies[18], after IDSA 2016 guidelines published[23], caspofungin was preferred over fluconazole. Amphotericin $\mathrm{B}$ and voriconazole were more used as target therapies.

The contributing factors of the finding of quite a low 28-day mortality rate for Candida BSI in patients from surgical wards compared to those of other wards need further study.

Since there was no significant difference in the 28-day mortality rate between the patients who received early appropriate $(26.8 \%)$ or targeted antifungal therapy (25.1\%), we further analyzed the demographic data, underlying diseases, and clinical features between these two groups. We found no significant differences between the two groups in age $(\mathrm{P}=0.33)$, sex $(\mathrm{P}=0.89)$, number of underlying diseases $(\mathrm{P}=0.32)$, number of severe clinical features $(\mathrm{P}=0.96)$, APACHE II score $(\mathrm{P}=0.072)$, or SOFA score $(\mathrm{P}=0.310)$. The choice of antifungal agents between these two groups was further analyzed. In the early appropriate antifungal therapy group, the rate of azoles use was $50.6 \%$, and the rate of echinocandin use was $30.3 \%$. In the targeted antifungal therapy group, the rate of azoles use was $65.4 \%$, and the rate of echinocandin use was $17.3 \%$. The rate of echinocandin use was lower in the targeted antifungal therapy group than in the early appropriate antifungal therapy group $(\mathrm{P}=0.038)$. Is the lower rate of echinocandin use in the targeted antifungal therapy group one of the reasons that caused no significant difference in mortality between the two groups? Many studies have confirmed the important role of echinocandin in antifungal therapy. Echinocandin has been recommended as a first-line antifungal agent since 2009[18], with fluconazole as an acceptable alternative for selected patients, reflecting the efficacy demonstrated by echinocandins and increasing resistance observed with fluconazole[34, 35]. Therefore, we believe that a lower rate of echinocandin use in the delayed antifungal 
Table 4 Multivariable Cox regression analysis for 393 Candida bloodstream infection episodes

\begin{tabular}{|c|c|c|c|c|c|}
\hline & \multirow{2}{*}{$\begin{array}{l}\text { Survival } \\
(n=281)\end{array}$} & \multicolumn{2}{|c|}{ 28-day outcome } & \multicolumn{2}{|c|}{ Multivariable analysis } \\
\hline & & $\begin{array}{l}\text { Death } \\
(n=112)\end{array}$ & P-value & HR $(95 \% \mathrm{Cl})$ & P-value \\
\hline Male & 216(76.9) & $76(67.9)$ & 0.065 & - & - \\
\hline Age, years & $55.2(19.5)$ & $63.6(16.2)$ & $<0.01$ & $1.025(1.013-1.037)$ & $<0.001$ \\
\hline Underlying disease & & & & - & - \\
\hline Solid tumor & $91(32.4)$ & $27(24.1)$ & 0.106 & - & - \\
\hline Hematologic malignancy & $32(11.4)$ & $16(14.3)$ & 0.428 & - & - \\
\hline Diabetes mellitus & $49(17.4)$ & $28(25)$ & 0.088 & - & - \\
\hline Chronic Cardiac disease & $72(25.6)$ & $52(46.4)$ & $<0.01$ & - & 0.105 \\
\hline Chronic Pulmonary disease & $34(12.1)$ & $18(16.1)$ & 0.294 & - & - \\
\hline Chronic renal failure & $20(7.1)$ & $22(19.6)$ & $<0.01$ & $2.018(1.234-3.299)$ & 0.005 \\
\hline Skin barrier compromised & $24(8.5)$ & $2(1.8)$ & 0.015 & - & 0.308 \\
\hline $\begin{array}{l}\text { Prior surgical intervention } \\
\text { (<1 month) }\end{array}$ & 182(64.8) & $62(55.4)$ & 0.083 & - & - \\
\hline Corticosteroid use & $40(14.2)$ & $14(12.5)$ & 0.652 & - & - \\
\hline $\begin{array}{l}\text { Prior antifungal agents use } \\
\text { (<6 month) }\end{array}$ & $64(22.8)$ & $24(21.4)$ & 0.772 & - & - \\
\hline Severity of clinical feature & & & & - & - \\
\hline Fever $\left(\mathrm{T}>38.2^{\circ} \mathrm{C}\right)$ & $220(78.3)$ & $89(79.5)$ & 0.798 & - & - \\
\hline Parenteral nutrition & $124(44.1)$ & $56(50)$ & 0.292 & - & - \\
\hline Mechanical ventilation & $89(31.7)$ & $58(51.8)$ & $<0.01$ & $1.950(1.307-2.912)$ & 0.001 \\
\hline Renal replacement therapy & $32(11.4)$ & $17(15.2)$ & 0.305 & - & - \\
\hline Central venous catheter & $227(80.8)$ & $89(79.5)$ & 0.766 & - & - \\
\hline Severe neutropenia & $24(8.5)$ & $18(16.1)$ & 0.029 & $4.347(2.462-7.675)$ & $<0.001$ \\
\hline Antifungal therapy & $224(74.9)$ & $75(25.1)$ & 0.007 & $0.502(0.294-0.857)$ & 0.006 \\
\hline No treatment & $57(60.6)$ & $37(39.4)$ & & & \\
\hline
\end{tabular}

Data were expressed as mean \pm SD for continuous variables and $\mathrm{n}(\%)$ for categorical variables

SD standard deviation

treatment group is unlikely to lead to a decrease in the 28-day mortality rate.

Based on the above analysis, we suggest that early antifungal therapy has no significant impact on the 28 -day mortality rate compared with targeted antifungal therapy. Many clinicians currently administer empirical antifungal agents, and our study results could be used to guide the clinical care of their patients. Overuse of antifungal drugs inevitably leads to a waste of medical resources and increased drug resistance [36]. Several studies support our findings [15-17] that the severity of illness (APACHE-II Score) affected short-term survival in patients with Candida infection, whereas the choice of initial antifungal agents did not affect shortterm survival. Trifi et al. revealed no beneficial impact of an empirical antifungal therapy on 28- day survival or in preventing the occurrence of candidemia in critically ill patients with non-neutropenic sepsis [37]. However, some studies differed in their findings on the timing of antifungal agents' use. Bassetti et al. showed that the use of antifungal agents within $48 \mathrm{~h}$ of obtaining a positive blood culture result is an independent protective factor against mortality during hospitalization [14]. Similarly, Tedeschi et al. showed that the administration of appropriate antifungal agents within $72 \mathrm{~h}$ after a positive blood culture is a protective factor for mortality during hospitalization [38]. Our results revealed similar risk factors (age, severe neutropenia, and mechanical ventilation) and protective factors (antifungal therapy) for 28-day mortality.

Although our data were collected from a large hospital in Shanghai, several limitations of this study should be taken into consideration. First, this was a retrospective study performed at a single center, which could lead to selection bias. Also, the antifungal results in our study were according to 2012 CLSI, rather than the 2017 CLSI guidelines on antifungal susceptibility M60. Further, Echinocandin susceptibility testing has not been carried out at our hospital, relevant clinical data could not be obtained. Moreover, the timing of CVC removal, 
laboratory tests such as PCT, CRP, and $(1,3)$ - $\beta$-D-glucan were not included for analysis because of missing data.

\section{Conclusion}

Our retrospective study findings showed an increased incidence of Candida BSI in the past 11 years in Shanghai. Although the percentage of non- C. albicans spp. has been increasing, C. albicans spp. remains the most frequently isolated species. The mortality of patients with Candida BSI was quite high (28.5\%), especially in internal medicine wards (37.2\%). Antifungal therapy improved the short-term survival of patients with Candida BSI. Whether preemptive antifungal therapy should be initiated or antifungal therapy should be initiated after the antifungal susceptibility test needs further discussion.

\begin{abstract}
Abbreviations
BSI: Bloodstream infection; Cl: Confidence interval; CLSI: Clinical and Laboratory Standards Institute; CVC: Central venous catheter; EUCAST: European Committee on Antimicrobial Susceptibility Testing; HR: Hazard ratio; HIV: Human immunodeficiency virus; ICU: Intensive care unit; IDSA: Infectious Diseases Society of America; SD: Standard deviation.
\end{abstract}

\section{Acknowledgements}

We thank Ms. Wen-Jing ZHENG to produce the massive time first to the article English grammar, the usage, and so on has carried on the revision.

\section{Authors' contributions}

ZTY, EZC, and EQM made substantial contributions to conception and design. $Z T Y, L Z H, Y J Z, L W, T X L Z$, and $X Y L$ participated in the acquisition of data. ZTY, YJZ, and YC drafted the manuscript. ZTY and EZC revised it critically. All authors read and approved the final manuscript.

\section{Funding}

This work was supported by the Program for Outstanding Medical Academic Leader, National Nature Science Foundation of China [81772107,81772040], Scientific and Technological Innovation Act Program of Science and Technology Commission of Shanghai Municipality [18411950900], Key Discipline Construction Project of Shanghai Municipal Commission of Health and Family Planning [2016ZB0206], Clinical Research Innovation Project of Shanghai Hospital Development Center [SHDC12017116] and Program of Shanghai Jiao Tong University School of Medicine [DLY201803].

\section{Availability of data and materials}

All data generated or analyzed during this study are included in this published article.

\section{Code availability}

Not applicable.

\section{Declarations}

\section{Ethics approval and consent to participate}

The study was approved by the local institutional review board (Ruijin Hospital, Shanghai Jiao Tong University, School of medicine).

\section{Consent for publication}

Not applicable.

\section{Competing Interests}

The authors declare that they have no competing interests.

\section{Author details}

${ }^{1}$ Department of Emergency, Ruijin Hospital, Shanghai Jiao Tong University School of Medicine, Shanghai 200025, China. ${ }^{2}$ Emergency Center, Suining Central Hospital, Suining 629000, Sichuan Province, China. ${ }^{3}$ Department of Geriatrics, Ruijin Hospital, Shanghai Jiao Tong University School of Medicine, Shanghai, China. ${ }^{4}$ Department of Cardiovascular Surgery, Ruijin Hospital, Shanghai Jiao Tong University School of Medicine, Shanghai, China. ${ }^{5}$ Department of Emergency, Ruijin North Hospital, Shanghai Jiao Tong University School of Medicine, Shanghai 201801, China. ${ }^{6}$ Department of Laboratory Medicine, Ruijin Hospital, Shanghai Jiao Tong University School of Medicine, Shanghai, China. ${ }^{7}$ Pôle Sino-Français de Recherches en Science du Vivant Et Génomique, Ruijin Hospital, Shanghai Jiao Tong University School of Medicine, Shanghai, China.

Received: 2 March 2021 Accepted: 5 May 2021

Published online: 13 May 2021

\section{References}

1. Gudlaugsson O, Gillespie S, Lee K, Vande Berg J, Hu J, Messer S, Herwaldt L, Pfaller M, Diekema D. Attributable mortality of nosocomial candidemia, revisited. Clin Infect Dis. 2003;37(9):1172-7.

2. Zaoutis TE, Argon J, Chu J, Berlin JA, Walsh TJ, Feudtner C. The epidemiology and attributable outcomes of candidemia in adults and children hospitalized in the United States: a propensity analysis. Clin Infect Dis. 2005;41(9):1232-9.

3. Falagas ME, Apostolou KE, Pappas VD. Attributable mortality of candidemia: a systematic review of matched cohort and case-control studies. Eur J Clin Microbiol Infect Dis. 2006;25(7):419-25.

4. Bitar D, Lortholary O, Le Strat Y, Nicolau J, Coignard B, Tattevin P, Che D, Dromer F. Population-based analysis of invasive fungal infections, France, 2001-2010. Emerg Infect Dis. 2014;20(7):1149-55.

5. Jia X, Li C, Cao J, Wu X, Zhang L. Clinical characteristics and predictors of mortality in patients with candidemia: a six-year retrospective study. Eur J Clin Microbiol Infect Dis. 2018;37(9):1717-24.

6. Marchetti O, Bille J, Fluckiger U, Eggimann P, Ruef C, Garbino J, Calandra T, Glauser M-P, Täuber MG, Pittet D, Switzerland FINo. Epidemiology of candidemia in Swiss Tertiary care hospitals: secular trends, 1991-2000. Clin Infect Dis. 2004;38(3):311-20.

7. Wisplinghoff H, Bischoff T, Tallent SM, Seifert H, Wenzel RP, Edmond MB. Nosocomial bloodstream infections in US hospitals: analysis of 24,179 cases from a prospective nationwide surveillance study. Clin Infect Dis. 2004;39(3):309-17.

8. Horn DL, Neofytos D, Anaissie EJ, Fishman JA, Steinbach WJ, Olyaei AJ, Marr KA, Pfaller MA, Chang CH, Webster KM. Epidemiology and outcomes of candidemia in 2019 patients: data from the prospective antifungal therapy alliance registry. Clin Infect Dis. 2009;48(12):1695-703.

9. Wisplinghoff H, Ebbers J, Geurtz L, Stefanik D, Major Y, Edmond MB, Wenzel RP, Seifert H. Nosocomial bloodstream infections due to Candida spp. in the USA: species distribution, clinical features and antifungal susceptibilities. Int J Antimicrob Agents. 2014;43(1):78-81.

10. Kim SH, Yoon YK, Kim MJ, Sohn JW. Clinical impact of time to positivity for Candida species on mortality in patients with candidaemia. J Antimicrob Chemother. 2013:68(12):2890-7.

11. Tiraboschi IN, Pozzi NC, Farias L, Garcia S, Fernandez NB. Epidemiology, species, antifungal resistance and outcome of candidemia in a university hospital in Buenos Aires, Argentina for 16 years. Revista chilena de infectologia : organo oficial de la Sociedad Chilena de Infectologia. 2017;34(5):431-40.

12. Bassetti M, Peghin M, Timsit JF. The current treatment landscape: candidiasis. J Antimicrob Chemother. 2016;71(suppl2):ii13-22.

13. Murri R, Giovannenze F, Camici M, Torelli R, Ventura G, Scoppettuolo G, Sanguinetti M, Cauda R, Fantoni M. Systematic clinical management of patients with candidemia improves survival. J Infect. 2018;77(2):145-50.

14. Bassetti M, Molinari MP, Mussap M, Viscoli C, Righi E. Candidaemia in internal medicine departments: the burden of a rising problem. Clin Microbiol Infect. 2013;19(6):E281-4. 
15. Parkins MD, Sabuda DM, Elsayed S, Laupland KB. Adequacy of empirical antifungal therapy and effect on outcome among patients with invasive Candida species infections. J Antimicrob Chemother. 2007;60(3):613-8.

16. Taur Y, Cohen N, Dubnow S, Paskovaty A, Seo SK. Effect of antifungal therapy timing on mortality in cancer patients with candidemia. Antimicrob Agents Chemother. 2010;54(1):184-90.

17. Grim SA, Berger K, Teng C, Gupta S, Layden JE, Janda WM, Clark NM. Timing of susceptibility-based antifungal drug administration in patients with Candida bloodstream infection: correlation with outcomes. J Antimicrob Chemother. 2011;67(3):707-14.

18. Pappas PG, Kauffman CA, Andes D, Benjamin DK Jr, Calandra TF, Edwards JE Jr, Filler SG, Fisher JF, Kullberg B-J, Zeichner LO, Reboli AC, Rex JH, Walsh TJ, Sobe JD. Clinical practice guidelines for the management candidiasis: 2009 update by the infectious diseases society of America. Clin Infect Dis. 2009;48(5):503-35.

19. Gavazzi G, Mallaret MR, Couturier P, Iffenecker A, Franco A. Bloodstream infection: differences between young-old, old, and old-old patients. J Am Geriatr Soc. 2002;50(10):1667-73.

20. Blot S, Cankurtaran M, Petrovic M, Vandijck D, Lizy C, Decruyenaere J, Danneels C, Vandewoude K, Piette A, Vershraegen G, Van Den Noortgate N, Peleman R, Dirk V. Epidemiology and outcome of nosocomial bloodstream infection in elderly critically ill patients: a comparison between middle-aged, old, and very old patients. Crit Care Med. 2009:37(5):1634-41

21. Li F, Wu L, Cao B, Zhang Y, Li X, Liu Y. Surveillance of the prevalence, antibiotic susceptibility, and genotypic characterization of invasive candidiasis in a teaching hospital in China between 2006 to 2011. BMC Infect Dis. 2013;13:353.

22. Zhang L, Wang H, Xiao M, Kudinha T, Mao LL, Zhao HR, Kong F, Xu YC. The widely used ATB FUNGUS 3 automated readings in China and its misleading high MICs of Candida spp. to azoles: challenges for developing countries' clinical microbiology labs. PLoS ONE. 2014;9(12):e114004.

23. Pappas PG, Kauffman CA, Andes DR, Clancy CJ, Marr KA, OstroskyZeichner L, Reboli AC, Schuster MG, Vazquez JA, Walsh TJ, Zaoutis TE, Sobel JD. Clinical practice guideline for the management of candidiasis: 2016 update by the infectious diseases society of America. Clin Infect Dis. 2016;62(4):e1-50.

24. Clinical and Laboratory Standards Institute (CLSI). 2008. Reference method for broth dilution 386 antifungal susceptibility testing of yeasts; approved standard — third edition. CLSI document M27-A3. Wayne, Pennsylvania.

25. Ulu Kilic A, Alp E, Cevahir F, Ture Z, Yozgat N. Epidemiology and cost implications of candidemia, a 6-year analysis from a developing country. Mycoses. 2017;60(3):198-203.

26. Schroeder M, Weber T, Denker T, Winterland S, Wichmann D, Rohde H, Ozga A-K, Fischer M, Kluge S. Epidemiology, clinical characteristics, and outcome of candidemia in critically ill patients in Germany: a singlecenter retrospective 10-year analysis. Ann Intensive Care. 2020;10(1):1-12.

27. Ricotta EE, Lai YL, Babiker A, Strich JR, Kadri SS, Lionakis MS, Prevots DR, Adjemian J. Invasive candidiasis species distribution and trends, United States, 2009-2017. J Infect Dis. 2021;223(7):1295-302.
28. Pfaller MA, Andes DR, Diekema DJ, Horn DL, Reboli AC, Rotstein C, Franks B, Azie NE. Epidemiology and outcomes of invasive candidiasis due to non-albicans species of Candida in 2496 patients: data from the Prospective Antifungal Therapy (PATH) registry 2004-2008. PLoS ONE. 2014;9(7):e101510.

29. Maria S, Barnwal G, Kumar A, Mohan K, Vinod V, Varghese A, Biswas R. Species distribution and antifungal susceptibility among clinical isolates of Candida parapsilosis complex from India. Rev Iberoam Micol. 2018:35(3):147-50.

30. Neji S, Hadrich I, Trabelsi H, Abbes S, Cheikhrouhou F, Sellami H, Makni F, Ayadi A. Virulence factors, antifungal susceptibility and molecular mechanisms of azole resistance among Candida parapsilosis complex isolates recovered from clinical specimens. J Biomed Sci. 2017;24(1):67.

31. Zhang L, Wang H, Xiao M, Kudinha T, Mao LL, Zhao HR, Kong F, Xu YC. The widely used ATB FUNGUS 3 automated readings in China and its misleading high MICs of Candida spp. to azoles: challenges for developing countries' clinical microbiology labs. PLoS One. 2014;9(12):e114004.

32. Torres-Rodríguez JM, Alvarado-Ramírez E. In vitro susceptibilities to yeasts using the ATB FUNGUS 2 method, compared with Sensititre Yeast One and standard CLSI (NCCLS) M27-A2 methods. J Antimicrob Chemother. 2007;60(3):658-61.

33. Maldonado I, Fernández Canigia L, Vivot W, Domeca P, Davel G, Córdoba S. Evaluación de tres métodos para la detección de la sensibilidad in vitro de especies de Candida a los antifúngicos [Evaluation of three methods for in vitro detection of antifungal susceptibility of Candida species]. Rev Argent Microbiol. 2011;43(2):120-6.

34. Kett DH, Shorr AF, Reboli AC, Reisman AL, Biswas P, Schlamm HT. Anidulafungin compared with fluconazole in severely ill patients with candidemia and other forms of invasive candidiasis: support for the 2009 IDSA treatment guidelines for candidiasis. Crit Care (London, England). 2011;15(5):R253.

35. Cui N, Wang H, Qiu H, Li R, Liu D. Impact of initial empirical antifungal agents on the outcome of critically ill patients with invasive candidiasis: analysis of the China-SCAN study. Int J Antimicrob Agents. 2017:50(1):74-80.

36. Hamdy RF, Zaoutis TE, Seo SK. Antifungal stewardship considerations for adults and pediatrics. Virulence. 2017;8(6):658-72.

37. Trifi A, Abdellatif S, Daly F, Nasri R, Touil Y, Ben Lakhal S. Empiric antifungal and outcome in ICU patients. Tunis Med. 2019;97(4):579-87.

38. Tedeschi S, Tumietto F, Giannella M, Bartoletti M, Cristini F, Cioni G, Ambretti S, Carretto E, Sambri V, Sarti M, Viale P. Epidemiology and outcome of candidemia in internal medicine wards: A regional study in Italy. Eur J Intern Med. 2016;34:39-44.

\section{Publisher's Note}

Springer Nature remains neutral with regard to jurisdictional claims in published maps and institutional affiliations.
Ready to submit your research? Choose BMC and benefit from:

- fast, convenient online submission

- thorough peer review by experienced researchers in your field

- rapid publication on acceptance

- support for research data, including large and complex data types

- gold Open Access which fosters wider collaboration and increased citations

- maximum visibility for your research: over $100 \mathrm{M}$ website views per year

At BMC, research is always in progress.

Learn more biomedcentral.com/submissions 